\title{
Clinical efficacy of lumbar interbody fusion using a channel system combined with ozone therapy for the treatment of central-type L3-L4 lumbar disc herniation
}

\author{
YU WANG ${ }^{1}$, HONG SUN ${ }^{2}$ and SHUZHEN QIN ${ }^{3}$ \\ ${ }^{1}$ Department of Orthopedics, Rizhao Hospital of Traditional Chinese Medicine; ${ }^{2}$ Department of Anesthesiology, \\ People's Hospital of Rizhao; ${ }^{3}$ Operating Room, Rizhao Hospital of Traditional Chinese Medicine, \\ Rizhao, Shandong 276800, P.R. China
}

Received June 24, 2016; Accepted December 19, 2016

DOI: $10.3892 /$ etm.2016.4009

\begin{abstract}
The clinical efficacy of minimally invasive lumbar interbody fusion via the intervertebral foramen combined with ozone $\left(\mathrm{O}_{3}\right)$ therapy for the treatment of L3-L4 central-type lumbar disc herniation was explored. We recruited patients with sciatica who attended our hospital between July 2013 and October 2015 and underwent lumbar X-ray (anteroposterior and lateral view), lumbar flexion-extension radiographs, computed tomography, and magnetic resonance imaging after admission. Seventy-four patients with central-type lumbar disc herniation but no other complications were randomly selected and divided into the observation and control groups. The observation group comprised 37 patients treated with lumbar fusion using a channel system combined with $\mathrm{O}_{3}$ therapy, whereas the control group comprised 37 patients treated with lumbar fusion alone. The effects of the two therapies were evaluated using visual analog scale, Japanese Orthopaedic Association, and MacNab scores. There was no significant difference in scores between the two groups before surgery $(\mathrm{P}>0.05)$. The scores of the observation group after treatment were significantly lower than those before surgery and those of the control group $(\mathrm{P}<0.05)$. One patient in the observation group experienced no obvious improvement in symptoms after surgery, and two patients in the control group experienced postoperative recurrence; these three patients subsequently underwent laminectomy combined with planted bone fusion and internal fixation. There was no significant difference in total efficacy rates between the two groups $(P>0.05)$. Lumbar
\end{abstract}

Correspondence to: Dr Yu Wang, Department of Orthopedics, Rizhao Hospital of Traditional Chinese Medicine, 35 Wanghai Road, Rizhao, Shandong 276800, P.R. China

E-mail: wang_yu_111@163.com

Key words: lumbar fusion using a channel system, ozone, central-type lumbar disc herniation, lumbar spine, minimally invasive fusion using a channel system combined with $\mathrm{O}_{3}$ therapy for the treatment of L3-L4 central-type lumbar disc herniation is safe and effective. It has the advantages of reduced trauma, fewer complications, and rapid pain relief, and it promotes the recovery of lumbar function. Strict mastery of the surgical indications is key to the success of the procedure; however, it is worth expanding its use in the clinical setting.

\section{Introduction}

Central-type lumbar disc herniation has multiple clinical manifestations. Pain in the lower limbs can manifest unilaterally or bilaterally, and some patients exhibit cauda equina syndrome (1). Based on the condition of the patient, unilateral or bilateral laminectomy via fenestration is performed, whereas patients with concurrent spinal stenosis undergo total laminectomy. However, traditional open surgery has the disadvantages of severe trauma, significant blood loss, and injury to muscles, ligaments, fascia, and vertebrae. Some patients experience denervation after the muscles are stripped, instability of the lumbar spine, spinal canal stenosis caused by intraspinal scarring, and other sequelae. Simultaneously, the levels of stress hormones rise, inflammatory cytokines are released, and lipolysis and hyperglycemia occur; these inflammatory reactions in the injured tissue have adverse effects on the vital organs and on the immune system. Consequently, the postoperative recovery time is long, and there is a risk of continuing lumbar instability (2-4).

As a result of the rapid development of modern medical equipment, many new minimally invasive spinal surgery techniques have been introduced for the treatment of lumbar disc herniation (5). Lumbar fusion using a channel system combined with ozone $\left(\mathrm{O}_{3}\right)$ therapy combines traditional lumbar intervertebral disc treatment with a lateral approach and spinal endoscopy, and provides a new solution for central-type lumbar disc herniation with the potential to reduce trauma and complications. However, at present, the efficacy of this procedure for the treatment of L3-L4 lumbar disc herniation is unclear. In this study, we examined the efficacy of lumbar fusion using a channel system combined with $\mathrm{O}_{3}$ therapy for L3-L4 lumbar disc herniation. 


\section{Materials and methods}

Inclusion and exclusion criteria. The inclusion criteria were: i) Unilateral or bilateral lower extremity pain, with or without back pain; ii) significant nerve root symptoms: Computed tomography (CT) and magnetic resonance imaging (MRI) showed central-type intervertebral disc herniation, the imaging and clinical manifestations were consistent, and it was possible to locate the lesion in the responsible intervertebral disc; and iii) no improvement after normative and conservative treatment for six weeks. Patients were excluded if they had: i) Recurrence of symptoms already treated with surgery; ii) spinal fractures, deformities, inflammation, or tumors; iii) coagulopathies; and iv) mental disorders.

We recruited patients with lumbocrural pain who attended our hospital between July 2013 and October 2015 and who underwent lumbar X-ray (anteroposterior and lateral view), lumbar flexion-extension radiographs, CT, and MRI after admission. Thirty-seven patients with central-type lumbar disc herniation without other comorbidities were selected, comprising 22 men and 15 women aged 28-54 years, with an average age of $36.8 \pm 1.5$ years. These patients underwent nerve root stripping and decompression with percutaneous intervertebral foramen endoscopy. This study was approved by the Ethics Committee of Rizhao Hospital of Traditional Chinese Medicine and informed consent was provided by all patients.

Surgical technique. i) Preoperative preparation: Patients were placed in a prone position after general anesthesia with endotracheal intubation. W-shaped cushions were placed bilaterally under the anterior superior iliac spine and in front of the thorax. Both sides of the abdomen were raised, and both lower limbs were flexed. A C-arm-type X-ray was used to determine the surgical segment, and the body surface was marked to indicate the pedicle of the vertebral arch. Iodine disinfection was performed, the iodine was removed with alcohol, and the drape was then put into position.

ii) Surgical procedures: The asymptomatic side, or the side with the mildest symptoms, was fixed, and a longitudinal incision of a suitable size was made over the site where the pedicles connected to the vertebral arch; the skin, subcutaneous tissue, and lumbodorsal fascia were then cut. A MASTQuadrant expansion pipe was progressively inserted into the gap between the multifidus and longissimus muscles after blunt dissection with a dilator, and then a quadrant dilator was used to expand the surgical incision at the end to form a truncated, cone-shaped channel with a narrow top and a wide bottom. The surgical segment and position of the MASTQuadrant pipeline were confirmed by fluoroscopy, after which the light source and free arm were connected. Using an electric knife, the facet joints and transverse process of the surgical segment were fully exposed to determine the entry point of the surgical segment at the vertebral pedicle. The cortex was enlarged, and a passage was drilled from the vertebral pedicle to the centrum. The bony structure surrounding the passage was evaluated using a probe, a positioning pin was placed, and C-arm fluoroscopy was used to determine the location of the positioning pin.

Next, the positioning pin was removed, and then a universal pedicle screw of matching size was placed into the bony passage. An iron rod of matching size was selected and prebent: This connecting rod was installed, the vertebral clearance was confirmed to be properly obstructed after the nut was put on, and the nut was temporarily tightened. The side with developing or severe symptoms was treated using the same method as for the side that was decompressed: A work pipe was established, a light source was connected, the vertebral plate and facet joint were fully exposed, a screw channel was drilled, a positioning needle was inserted, the position of the pedicle screw channel was confirmed to be satisfactory by fluoroscopy, the positioning needle was removed, and then the channel entry was closed with bone wax.

An electric knife and a bipolar cauterizer were used to cauterize the interlaminar soft tissue of the vertebral plate during surgery to prevent bleeding. The intervertebral space of the surgical segment was exposed via curette cleaning of the vertebral plate. Next, the head of the vertebral plate was resected to the origin of the ligamentum flavum, the head of the caudal part of the vertebral plate was resected, and the end of the ligamentum flavum was exposed. The nerve root was stripped with a nerve hook to reveal the yellow ligament along the central fissure, which was cleared, and the dura and nerve root were exposed. A nerve hook was then used to pull the nerve root. The fiber ring was cut annularly using a knife, and the protruding lumbar disc was removed with the small nucleus pulposus. If patients had concurrent significant bilateral nerve root injury, then bilateral decompression was performed using the same method; if the nerve root canal exhibited stenosis, it was expanded.

Nerve root stripping using a nerve hook permitted careful exploration of the nerve root, and allowed us to determine whether the decompression of the nerve root was sufficient and whether the nerve root canal was unobstructed. A curette was used to dispose of the cartilage endplate, and the endplate tissue was removed with the nucleus pulposus. The size of the fusion device was determined, and autologous bone combined with NanoBone was placed into the fusion device. The remaining broken bone was implanted in the front edge of the intervertebral space and placed in the intervertebral fusion device after compression. Fluoroscopy was repeated to confirm that the intervertebral fusion device was in the correct position. In the observation group, 5-15 $\mathrm{ml}$ of $\mathrm{O}_{3}$ was injected into the intervertebral disc; the control group was not injected with $\mathrm{O}_{3}$. A universal pedicle screw was placed on the decompression side, and fluoroscopy was used to confirm its position. The nut was loosened on the fixed side, and the nuts were then compressed and tightened on both sides. The working pipeline was removed, the bleeding was fully stopped, the intervertebral space was washed, a negative pressure drainage tube was placed after irrigation with physiologic saline, and the wound was closed layer by layer. Sterile dressings were used to cover the wound, and the operation was completed (6-8) (Fig. 1).

Evaluation of efficacy. Evaluation of visual analog scale (VAS), Japanese Orthopaedic Association (JOA), and MacNab scores was performed. All patients were followed up for at least one year by telephone and as outpatients. VAS, JOA, and MacNab scores for pain in the waist and legs were recorded preoperatively and at 3, 6, 12 and 18 months postoperatively (2). 
$\mathbf{A}$

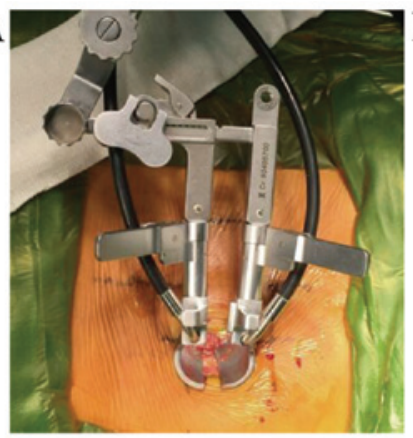

D

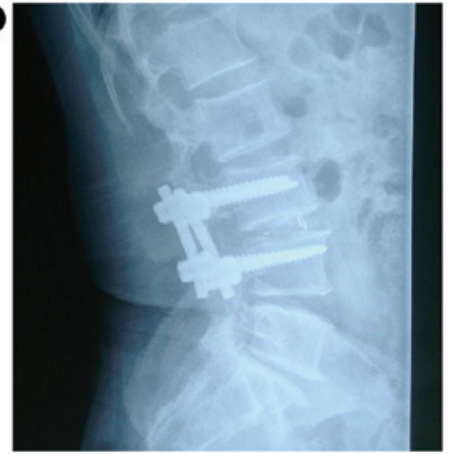

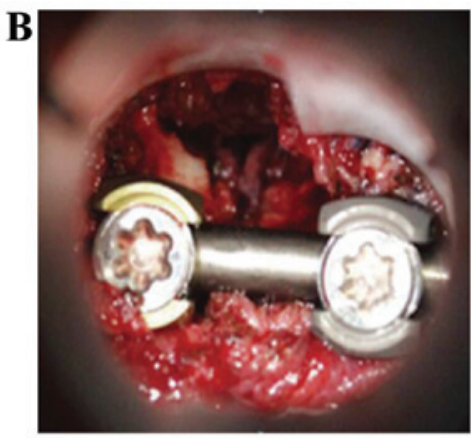

$\mathbf{E}$

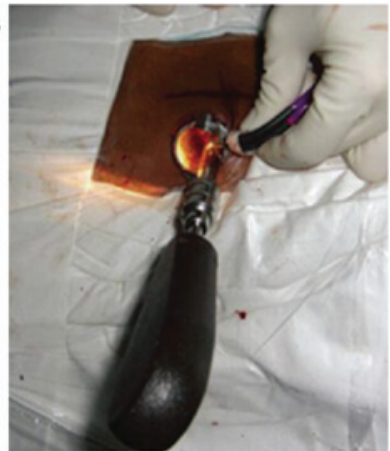

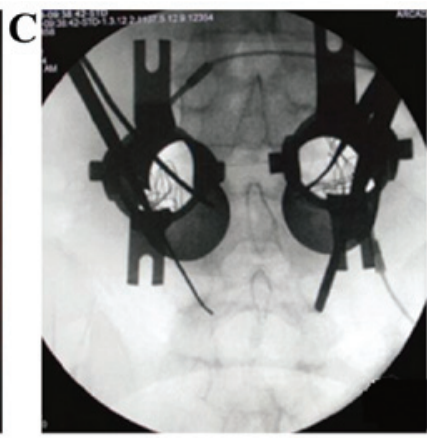

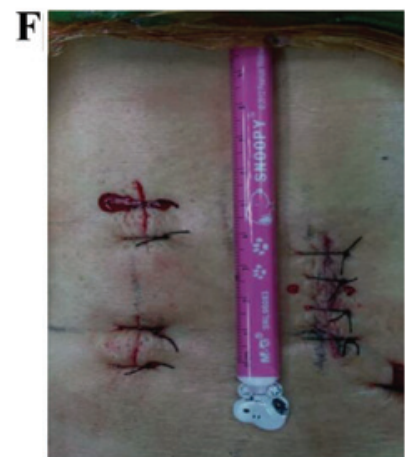

Figure 1. Surgical procedures. (A) Work channel placement; (B) pedicle screw placement under erthyphoria; (C) internal fixation of pedicle screws through bilateral channels; (D) mixed internal fixation of the unilateral channel; (E) incision made for the mixed internal fixation; (F) postoperative incision suture.

Table I. Basic characteristics of the 74 patients with L3-L4 lumbar disc herniation.

\begin{tabular}{|c|c|c|c|c|c|c|c|}
\hline Groups & Age (years) & $\begin{array}{c}\text { Course of } \\
\text { disease (years) }\end{array}$ & MAP (mmHg) & $\begin{array}{c}\text { Bleeding } \\
\text { volume }(\mathrm{ml})\end{array}$ & $\begin{array}{c}\text { Clear volume of } \\
\text { intervertebral disc }(\mathrm{ml})\end{array}$ & $\begin{array}{l}\text { Operation } \\
\text { time }(\min )\end{array}$ & BMI $\left(\mathrm{kg} / \mathrm{m}^{2}\right)$ \\
\hline Observation & $45.3 \pm 5.5$ & $1.33 \pm 0.44$ & $102.8 \pm 22.9$ & $112.4 \pm 10.5$ & $9.8 \pm 1.6$ & $109.4 \pm 10.2$ & $18.8 \pm 1.24$ \\
\hline Control & $39.8 \pm 4.7$ & $1.27 \pm 0.89$ & $108.4 \pm 18.7$ & $109.7 \pm 9.4$ & $10.2 \pm 1.7$ & $112.4 \pm 11.3$ & $19.7 \pm 0.87$ \\
\hline t-value & 0.98 & 1.22 & 0.78 & 0.33 & 0.98 & 0.37 & 0.87 \\
\hline P-value & $>0.05$ & $>0.05$ & $>0.05$ & 0.71 & 0.14 & 0.42 & $>0.05$ \\
\hline
\end{tabular}

BMI, body mass index; MAP, mean arterial pressure.

Statistical analysis. The statistical software program SPSS 13.0 (SPSS Inc., Chicago, IL, USA) was used to analyze the data. VAS, JOA, and MacNab scores were analyzed by paired t-test. A $\mathrm{P}<0.05$ was considered to indicate a statistically significant difference.

\section{Results}

Comparison of the basic characteristics of 37 patients with lumbar disc herniation. Thirty-seven patients were successfully operated on and followed up for 0-18 months (average, 12.1 months). Preoperative and postoperative imaging and intraoperative data were recorded. There was no significant difference in baseline data between the two groups $(\mathrm{P}>0.05)$ (Table I).

Comparison of therapeutic effects. The preoperative scores for all showed no significant differences between the two groups (P>0.05). After treatment, the scores for all items for patients in the observation group were significantly lower than the preoperative scores, and significantly lower compared with the control group $(\mathrm{P}<0.05)$. One patient in the observation group did not experience any obvious improvement in symptoms after surgery, and two patients in the control group experienced postoperative recurrence; these patients then underwent laminectomy combined with planted bone fusion and internal fixation. There was no significant difference in total efficacy rates between the two groups $(\mathrm{P}>0.05)$ (Table II).

MacNab scores. The MacNab scores after treatment for the patients in the two groups were analyzed. The overall score of the observation group was significantly higher than that of the control group $\left(\chi^{2}=42.87\right.$, Chi-square test $)$, and this difference was statistically significant $(\mathrm{P}<0.05)$ (Table III).

\section{Discussion}

Lumbar disc herniation refers to a set of clinical symptoms produced by the nucleus pulposus protruding to the rear of the spine or into the interlaminar space. Degeneration of the 
Table II. Effect of lumbar interbody fusion under channel.

\begin{tabular}{lccccrrr}
\hline Time & Cases (n) & $\begin{array}{c}\text { VAS scores of } \\
\text { lower limbs }\end{array}$ & t-value & P-value & JOA scores & t-value & P-value \\
\hline Pre-operation & 37 & $7.85 \pm 0.62$ & & & $11.6 \pm 0.62$ & \\
Post-operation, 3 months & 37 & $2.18 \pm 0.25$ & 72.38 & $<0.001$ & $19.4 \pm 0.98$ & 42.8 & $<0.001$ \\
Post-operation, 1 year & 37 & $1.96 \pm 0.48$ & 148.3 & $<0.001$ & $20.1 \pm 0.72$ & 50.7 & $<0.001$ \\
Last follow-up & 37 & $2.02 \pm 0.13$ & 59.1 & $<0.001$ & $20.2 \pm 0.26$ & 135.3 & $<0.001$ \\
\hline
\end{tabular}

VAS, visual analog scale; JOA, Japanese Orthopaedic Association.

Table III. MacNab scores of patients in two groups before and after treatment.

\begin{tabular}{|c|c|c|c|c|c|}
\hline Groups & Time & Excellent & Good & Tolerable & Poor \\
\hline \multirow[t]{4}{*}{ Observation } & Pre-operation & 0 & 0 & 2 & 35 \\
\hline & $\begin{array}{l}\text { Post-operation, } \\
3 \text { months }\end{array}$ & 27 & 3 & 7 & 1 \\
\hline & $\begin{array}{l}\text { Post-operation, } \\
1 \text { year }\end{array}$ & 30 & 2 & 5 & 0 \\
\hline & Last follow-up & 32 & 1 & 3 & 1 \\
\hline \multirow[t]{6}{*}{ Control } & Pre-operation & 0 & 0 & 12 & 25 \\
\hline & $\begin{array}{l}\text { Post-operation, } \\
3 \text { months }\end{array}$ & 18 & 4 & 4 & 1 \\
\hline & $\begin{array}{l}\text { Post-operation, } \\
1 \text { year }\end{array}$ & 20 & 8 & 5 & 4 \\
\hline & Last follow-up & 24 & 9 & 1 & 3 \\
\hline & $\chi^{2}$ value & 42.87 & & & \\
\hline & $\mathrm{P}$-value & $<0.001$ & & & \\
\hline
\end{tabular}

lumbar intervertebral disc and rupture of the fibrous ring results in the irritation and compression of adjacent tissues, leading to pain in the waist, unilateral or bilateral numbness in the lower limbs, and other clinical symptoms. The incidence of L3-L5 and L5-S1 lumbar disc herniation is highest, accounting for $\sim 95 \%$ of cases (1-3). Therefore, the condition is a serious threat to the health of elderly individuals. Lumbar disc herniation caused by intervertebral disc degeneration is mainly due to increased negative pressure, extra strain exerted by pregnancy, incorrect lumbar posture, sudden weight-bearing, extreme cold, and excess moisture $(2,4)$. The condition can be divided into three types based on the position of the protruding intervertebral disc: posterolateral protrusion; protrusion into the intervertebral foramen; and central protrusion (9).

Central lumbar disc herniation $(\mathrm{CLDH})$ refers to protruding and herniated disc tissue in the anterior central position. The posterolateral part of the lumbar intervertebral disk is its weakest point; thus, posterolateral protrusion is the most common type of lumbar disc herniation. However, CLDH is not uncommon, and affects 5.4-33.4\% of patients with herniation (10). Surgical treatment should be used in patients if conservative treatment is ineffective $(10,11)$. The traditional posterior median approach for lumbar interbody fusion fixes the pedicle of the vertebral arch internally with a screw to relieve compression of the nerve root, and effectively improves patients' clinical manifestations. However, because this surgery destroys the stable structure of the lumbar spine, this single operation cannot revert the waist structure to its normal state. Good posture and mechanical function of the body must be maintained after this operation.

$\mathrm{O}_{3}$ is a strong oxidizing agent. It causes water-loss atrophy of the nucleus pulposus by destroying the proteins and polysaccharides in the stroma of the nucleus pulposus, relieving stress on the nerve root caused by protrusion of the nucleus pulposus. $\mathrm{O}_{3}$ can also destroy nucleus pulposus cells, cause production of polysaccharide in the nucleus pulposus, and decrease protein secretion. In addition, treatment with $\mathrm{O}_{3}$ promotes the dissipation of inflammation and reduces the release of inflammatory factors. These processes aid in the resolution of lumbar disc herniation by (12-14): i) Influencing cytokine antagonists, and/or inhibiting autoimmunity cytokines such as interleukin (IL)-10 and transforming growth factor (TGF)- $\beta 1$; ii) causing the overexpression of antioxidants to neutralize excessive reaction products; and iii) preventing vascular endothelial cells from producing platelet-derived growth factor, which causes blood vessels to dilate, exacerbating inflammation.

The risk of postoperative infection is greatly reduced, because $\mathrm{O}_{3}$ is a disinfectant. Ordinarily, an increase in the release of pain-associated inflammatory cytokines, such as IL-6, IL-10, and TGF- $\beta 1$, will cause further inflammatory cell infiltration; thus, postoperative pain is perpetuated, and may even necessitate secondary surgery. Therefore, lumbar $\mathrm{O}_{3}$ therapy combined with lumbar spinal stabilization via channel fusion can significantly ameliorate intervertebral disc inflammation and relieve pain.

In addition to the treatment of lumbar disc herniation, Duymus et al (15) reported that the use of $\mathrm{O}_{3}$ effectively improves the clinical symptoms of osteoarthritis. This conclusion was also confirmed in animal experiments. After treating C57BL6/N mice with $\mathrm{O}_{3}$ and $\mathrm{O}_{2}$, their visceral pain disappeared (16). Intra-articular injection of $\mathrm{O}_{3}$ has also been shown have good therapeutic effects in the treatment of osteoarthritis of the knee (17). However, $\mathrm{O}_{3}$ treatment has certain limitations, as the scope of its application is narrow, only a single injection of no more than $20 \mathrm{ml}$ is typically possible, and it is predominantly effective for mild intervertebral disc protrusion. It does not have a significant curative effect on moderate or severe intervertebral disc protrusion, and it cannot directly eliminate the nucleus pulposus that compresses the nerve tissue. Also, 
patients with cervical disc herniation who receive $\mathrm{O}_{3}$ therapy can experience thunderclap headache caused by unexpected epidural puncture (18). Despite this, we believe that surgery combined with $\mathrm{O}_{3}$ therapy can significantly improve the clinical symptoms of patients with lumbar disc herniation and effectively reduce inflammation with good therapeutic efficacy (19-21).

In conclusion, lumbar fusion using a channel system combined with $\mathrm{O}_{3}$ therapy for the treatment of L3-L4 CLDH is safe and effective. It has the advantages of reduced trauma, fewer complications, and rapid pain relief, and it promotes the recovery of lumbar function. Strict mastery surgical indications for this method is key to the success of the operation and its curative effect; nevertheless, the procedure is worth performing more regularly in the clinical setting.

\section{References}

1. Kitagawa Y, Sairyo K, Shibuya I, Kitahama Y, Kanamori Y, Koga S, Matsumoto H, Sumita T, Yamada A and Dezawa A: Minimally invasive and simultaneous removal of herniated intracanal and extracanal lumbar nucleus pulposus with a percutaneous spinal endoscope. Asian J Endosc Surg 5: 183-186, 2012.

2. Rouben D, Casnellie M and Ferguson M: Long-term durability of minimal invasive posterior transforaminal lumbar interbody fusion: a clinical and radiographic follow-up. J Spinal Disord Tech 24: 288-296, 2011.

3. Yoon MA, Hong SJ, Kang CH, Ahn KS and Kim BH: T1rho and T2 mapping of lumbar intervertebral disc: correlation with degeneration and morphologic changes in different disc regions. Magn Reson Imaging 34: 932-939, 2016.

4. Lee JH and Lee SH: Clinical and radiological characteristics of lumbosacral lateral disc herniation in comparison with those of medial disc herniation. Medicine (Baltimore) 95: e2733, 2016.

5. Huang W, Han Z, Liu J, Yu L and Yu X: Risk factors for recurrent lumbar disc herniation: a systematic review and meta-analysis. Medicine (Baltimore) 95: e2378, 2016.

6. Kim TW, Oh CH, Shim YS, Yoon SH, Park HC and Park CO: Psychopathological influence of lumbar disc herniation in male adolescent. Yonsei Med J 54: 813-818, 2013.

7. Paik HK, Oh CH, Choi K, Kim CE, Yoon SH and Chung J: Influence of history of brain disease or brain trauma on psychopathological abnormality in young male in Korea: analysis of multiphasic personal inventory test. J Korean Neurosurg Soc 50: 114-118, 2011.

8. Oh CH, Lim HK, Chung J, Yoon SH, Park HC and Park CO: The psychopathological influence of congenital heart disease in Korean male adolescents: an analysis of multiphasic personal inventory test results. Yonsei Med J 53: 1107-1112, 2012.
9. Kim DK, Oh CH, Lee MS, Yoon SH, Park HC and Park CO: Prevalence of lumbar disc herniation in adolescent males in Seoul, Korea: prevalence of adolescent LDH in Seoul, Korea. Korean J Spine 8: 261-266, 2011.

10. Yue B, Chen B, Zou YW, Xi YM, Ren XF, Xiang HF, Hu YG and Zhang G: Thoracic intervertebral disc calcification and herniation in adults: a report of two cases. Eur Spine J 25 (Suppl 1): 118-123, 2016.

11. Xu F, Yin Y, Liu C, Yi Y, Yu J, Kou D and Wang S: Correlations of $\mathrm{O}_{3}$ therapeutic targets and imaging localization in lumbar intervertebral disc protrusion. Int J Clin Exp Med 8: 9283-9290, 2015.

12. Chen H, Yu B, Lu C and Lin Q: The effect of intra-articular injection of different concentrations of ozone on the level of TNF- $\alpha$, TNF-R1, and TNF-R2 in rats with rheumatoid arthritis Rheumatol Int 33: 1223-1227, 2013.

13. Dagistan Y, Cukur S, Dagistan E and Gezici AR: Importance of IL-6, MMP-1, IGF-1, and BAX levels in lumbar herniated disks and posterior longitudinal ligament in patients with sciatic pain. World Neurosurg 84: 1739-1746, 2015.

14. Elvis AM and Ekta JS: Ozone therapy: a clinical review. J Nat Sci Biol Med 2: 66-70, 2011.

15. Duymus TM, Mutlu S, Dernek B, Komur B, Aydogmus S and Kesiktas FN: Choice of intra-articular injection in treatment of knee osteoarthritis: platelet-rich plasma, hyaluronic acid or ozone options. Knee Surg Sports Traumatol Arthrosc: Apr 7, 2016 (Epub ahead of print).

16. Nellensteijn J, Ostelo R, Bartels R, Peul W, van Royen B and van Tulder M: Transforaminal endoscopic surgery for symptomatic lumbar disc herniations: a systematic review of the literature. Eur Spine 19: 181-204, 2010.

17. Hashemi M, Jalili P, Mennati S, Koosha A, Rohanifar R, Madadi F, Razavi SS and Taheri F: The effects of prolotherapy with hypertonic dextrose versus prolozone (intraarticular ozone) in patients with knee osteoarthritis. Anesth Pain Med 5: e27585, 2015.

18. Liu H, Wang Y, An JX, Williams JP and Cope DK: Thunderclap headache caused by an inadvertent epidural puncture during oxygen-ozone therapy for patient with cervical disc herniation. Chin Med J (Engl) 129: 498-499, 2016.

19. Cho JY, Lee SH and Lee HY: Prevention of development of postoperative dysesthesia in transforaminal percutaneous endoscopic lumbar discectomy for intracanalicular lumbar disc herniation: floating retraction technique. Minim Invasive Neurosurg 54: 214-218, 2011.

20. Celikoglu E, Kiraz I, Is M, Cecen A and Ramazanoğlu A: The surgical treatment of far lateral lumbar disc herniation: 33 cases. Acta Orthop Belg 80: 468-476, 2014.

21. Kasai Y, Inaba T, Kato T, Matsumura Y, Akeda K and Uchida A: Biomechanical study of the lumbar spine using a unilateral pedicle screw fixation system. J Clin Neurosci 17: 364-367, 2010. 